\title{
Technical development at the 2TRAIN project
}

\author{
J. M. Mera ${ }^{1}$, L. Brue ${ }^{2}$, M. Hintenender ${ }^{3}$, A. Gil ${ }^{1} \&$ L. M. Gutierrez ${ }^{1}$ \\ ${ }^{I}$ CITEF, Universidad Politécnica de Madrid, Spain \\ ${ }^{2}$ CORYS TESS, France \\ ${ }^{3} \mathrm{KMW}$, Germany
}

\section{Abstract}

The establishment of safe, competitive, and interoperable railways in Europe requires giving particular attention to the formation and qualification of train drivers.

Moreover, as Europe grows together and cross-border operations increase there is a strong need to harmonise and coordinate the education of train drivers concerning general driving and operational abilities as well as particular crisis management competencies. Important benefits can be obtained by the use of computer-based systems and simulators in training, with the usage of these technologies training sessions can be composed of different technical failures and hazardous operational situations of which many cannot be trained in reality, furthermore, it is possible to replicate scenarios at any given time.

The 2TRAIN project (Training of Train Drivers in Safety Relevant Issues with Validated and Integrated Computer Based Technology), financed by the 6FP of the European Union, aims to develop European best tools and guidelines for efficient, safety enhancing and cost-effective use of modern technologies for training as well as for ongoing competence and performance assessment.

The starting point of 2TRAIN was a benchmarking of training technology, contents and models already in use in Europe. In addition, to reach a harmonisation of today's training technologies and to allow a standardised driver evaluation, a set of common software modules are currently being developed, the first of these modules is the common data simulation interface, this interface will be implemented into three simulator pilots as the linking point between existing technologies and new modules.

In the second step common training simulation scenarios will be created, five European operators will use them for training experienced and inexperienced drivers. The trainees' actual behaviour will be compared to predefined target behaviour by the virtual instructor module in real time.

This target behaviour will be defined by a rule-based expert system, the information for building up this expert system will be derived from the rulebooks and directives of the railway undertakings, but "real instructors" will be able to adjust and create new rules.

The results of the assessment will be stored in an assessment database that could be used as a base for the development of further add-on systems.

The quality of 2TRAIN is ensured by a close collaboration between operational railway companies (CD, DB, Metro de Madrid and SNCF), simulator developers (CITEF, CORYS T.E.S.S. and KMW) transport institutions (IJP, RTI), universities (IZVW, UP) and by the strong support from a user group that consists of further European stakeholders.

Keywords: computer-based training, driving simulation. 


\section{State of railway simulation in Europe}

In the first 2TRAIN project workpackage a series of studies were undertaken on the methods, technologies and contents used by different European operators for training train drivers and were divided into two stages. Two reports were made in the first stage; the first [1] was focussed on a review of both European and world Standards concerning the application of computer technologies to training (SCORM, HLA...); the second report [2] was based on the study of driver competencies as well as on the typical training contents that are usually applied in railway companies.

In the second stage it was attempted to conduct a direct review of the technologies and contents applied in European companies. To this end questionnaires were sent to over 40 railway operators, and surveys and face-toface interviews were carried out with 18 of the most representative companies in Europe.

The results obtained after analysing the interviews were set out in two reports. The first of these, entitled "Benchmarking report, Training tools and technology" [3] is focussed towards a study of the use of computer tools in driver training. The second of the reports is entitled "Benchmarking report, Training contents and training modules" [4] and analyses both the form and content of the different types of training currently used by European operators.

As a result of the study on the technical side, European train simulators often consist of a full cab replica (maybe including a motion system) with real controls, high level visualization system and a realistic train behaviour model. In these simulators one trainee is trained whilst one instructor is supervising him. The training contents are explained in detail in the training contents and training models report, but usually the trainee is trained in general circulation, signalization and train management. Although this could be considered the "typical simulator", almost every company has a specific customization.

Also, the learning objectives of these companies are quite different; most of the operators prefer individualized attention, but others (for example some metro operators) have chosen an integrated training simulation centre where drivers, signallers and station managers can be trained together. The actions of each one of these roles affect (if desired) the simulation scenario of the others. So, definitely the objective of these simulation centres is to train all these roles and thorough cooperative training exercises to teach them to work together.

As a consequence of these different training procedures, operators have different technical requirements; for some companies a real cab replica simulator is fundamental, whereas others prefer smaller replicas, small driver desks or even software interface simulators.

The presence or absence of a motion system is also a consequence of the different training procedures. Some companies, mainly main line operators in the North of Europe think that a realistic motion system is fundamental to reach a high immersion level of experienced train drivers, therefore these companies have simulators with motion systems. On the other hand, some companies, 
usually metro operators, think that these systems are not worthwhile for their training methods; hence, these companies have not got motion systems.

With regard to the simulated train, some driving simulators are rolling stock specific; they simulate a specific train while others are more general. The former are used to teach trainees how to drive a precise train whereas the latter are usually utilized to teach about driving regulation and signalization.

Finally, in relation to the opinion that operators have about their simulator, the best rated items are the motion systems (in simulators having such a feature) and the mathematical model of the train and the signalization model; on the contrary the final exercise report gets the lower marks. As one of the main objectives of the 2TRAIN project is to improve this report, we expect this evaluation to rise in the future.

\section{2Train overview}

As already explained, in recent years railway operators have been using computer tools (mainly simulators and CBTs) for driver training. But as this is a fairly recent and currently expanding field of application, each company uses these means in an individual rather than a standardised way.

The 2TRAIN project aims to establish a basis for initiating a harmonisation process for driver training in Europe including competencies and safety conditions.

Each European operator is subject to different laws, internal rules, signalling systems and in general, training structures. As a result of this diversity a total harmonisation of training and qualification technologies will be unattainable.

However, as Europe is increasing its cross-border operations there is a great need to harmonise and co-ordinate driver training regards driving skills and general abilities, as well as their competencies in crisis management in particular.

Important benefits can be obtained by the use of computer-based systems and simulators in training. With the usage of these technologies training sessions can be composed of different technical failures and hazardous operational situations, many of which cannot be trained in reality; furthermore, it is possible to replicate scenarios at any given time.

The starting point of 2TRAIN was a benchmarking of training technology, contents and models already in use in Europe. In addition, to reach a harmonisation of today's training technologies and to allow a standardised driver evaluation, a set of common software modules is currently being developed. The first of these modules is the common data interface, which will be implemented into three simulator pilots as the linking point between existing technologies and new modules.

In the second step common training simulation scenarios and CBT modules will be created. Four European operators will use them for training experienced and inexperienced drivers.

The trainees' actual behaviour will be compared to predefined target behaviour by the virtual instructor module in real time. This target behaviour will 
be defined by a rule-based expert system. The information for building up this expert system will be derived from the rulebooks and directives of the railway companies, but "real instructors" will be able to adjust rules and even create new rules. The results of the assessment will be stored in an assessment database that could be used as a base for the development of further add-on systems.

One of the 2TRAIN project's goals is also to develop European good practice guidelines for enhancing railway safety, the efficient use of training technologies, the improvement of competencies and the evaluation and assessment of the outcomes obtained.

\section{Developments}

As explained above, the development of the 2TRAIN software comprises five modules; the CDSI (common data simulation interface), the RBES (Rule-Based Expert System), the VI (virtual instructor), and the AssDB (Assessment Database).

In principle, the project is based on adding these modules to each of the simulators (or CBTs) avoiding, as far as possible, any need to make any changes to the software of the simulators themselves. Due to this fact, the VI will be run on a laptop beside the driver and the RBES on another computer next to the instructor.

The instructor, thanks to the RBES's graphic interface, will set out the national regulations together with those of the individual operator in a series of rules that will be saved to an XML format text file. These rules must only be created one single time although they can be modified whenever so wished.

When an exercise is launched, the VI will automatically load this file and ensure that it observes the rules written in it. Depending on how the rules are configured the VI itself may display alert messages to the driver or even show him more detailed help files.

In addition, as an aid to the actual instructor, the RBES possesses a graphic interface (GUI) where the status of the exercise is shown in real time, indicating, for example, the rules that are not being observed.

Once the exercise is completed, the VI generates a report in a table format (which in turn has an XML [5] format) and sends it to the AssDB. It will be finally the latter that generates a ready-to-print report of the exercise at the instructor's request.

It should be pointed out that on an internal level, the communication of variables between the VI, the RBES and the simulator itself is conducted by means of a new communication layer called CDSI and any communication between the VI and the AssDB is performed by means of an ADO [6].

Below is a more detailed description of each module's characteristics:

CDSI: This is the system for communicating variables and messages, developed by CORYS T.E.S.S. [7]; it is based on CORBA and makes communication possible between the different 2TRAIN modules (mainly VI and RBES) and the simulators themselves. 


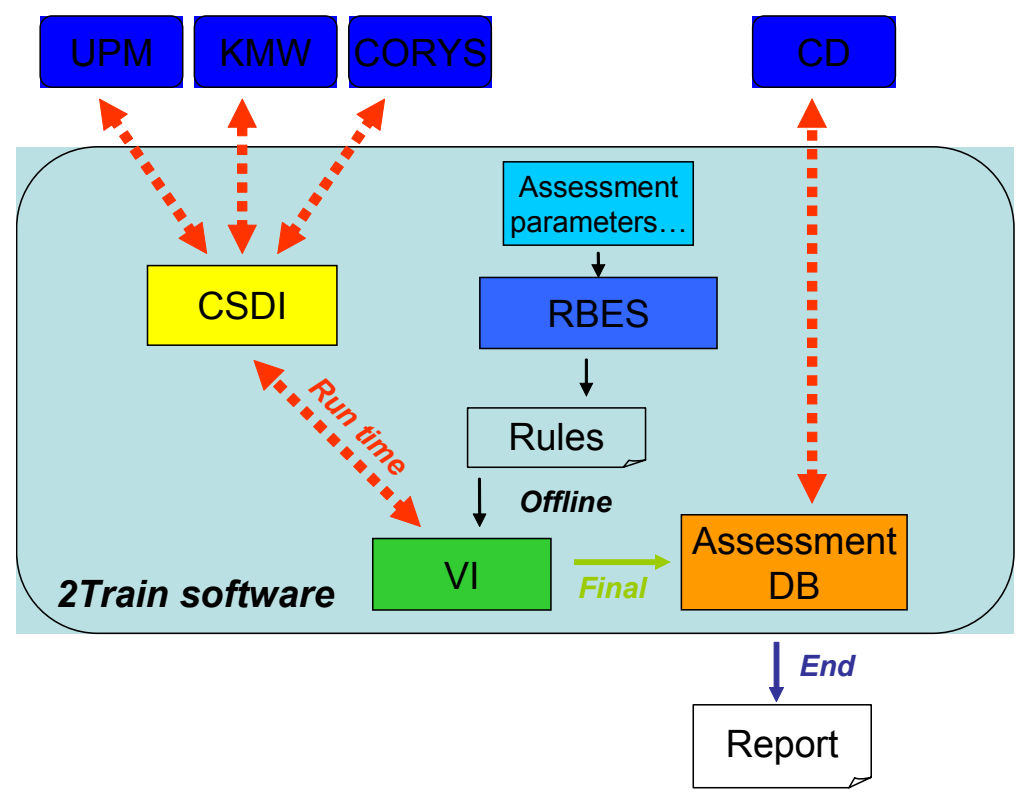

Figure 1.

The main functions of the CDSI are:

- To communicate the value of certain variables (the simplest example of a transmitted variable is the train speed)

- Message dispatch (among other things, the messages will be used to pause and re-start the simulator)

Depending to what extent they are used, two types of variables have been defined:

- variables common to all simulators: these are the variables implemented by all simulators; by means of these it is hoped to facilitate an interchange of rules between organisations. There is currently a preliminary version of this list of variables although its being increased in the future cannot be ruled out.

- variables specific to each simulator: these are conceived so that the circumstances specific to each simulator can be handled.

RBES: Also known as RBES (Rule Based Expert System); this is the expert system for creating rules which is being developed by KMW [8]. It has two different behaviours depending on the time it is run:

RBES Alone: An application is being developed by which instructors will be able to create rules. This application will come with a graphic interface that will help generate changes to rules at the click of a mouse.

Real Time RBES: Its mission is to send information from the real instructor about the situation of the exercise as well as to ask the instructor about specific driver actions or behaviours that cannot be assessed by computer. Examples of such behaviours may be assessing the attention paid while the exercise is being 
run or the clarity with which the trainee communicates with the control stations by radio-telephone.

AssDB: This is a database developed by UP [9], based on PostgreSQL [10], which will store the information concerning each exercise. Any communication between it and the VI is conducted by means of ADO.

The report tables generated by the VI are stored in this database, but in addition the value of the variables chosen by the instructors for subsequent use or review can also be stored.

Besides storing the data referring to the exercises, the AssDB will also come with a graphic interface (web server-based) by means of which the instructors will be able to generate reports from the saved data in each simulation. With this graphic interface it will also be possible to compare parameters between different simulators.

VI: The mission of this system, developed by CITEF [11], is to evaluate the rules in real time as well as to execute actions associated with them when necessary; for example, the VI is capable of displaying help to the driver when he has committed some error.

\section{Rule and action-based system}

As already stated, assessing driver behaviour in respect of ideal behaviour is done by using a series of rules. In principle, there will be a rule for each concept that it is wished to assess. One example of a rule may be if the driver maintains an adequate speed and another if he brakes appropriately when faced with a red signal.

The set of generic instructions entrusted with assessing a concept is called RuleTemplate. When these instructions are applied to a specific situation an instance of the RuleTemplate is created called RuleInstance.

In line with the above examples, we can only have a single RuleInstance for speed, but for a halt at a signal, there will be as many RuleInstances as there are signals on the line, since each of them will have its own particular starting and stopping point.

The virtual instructor may have as many RuleInstances loaded in its memory as are needed to assess the different driving aspects (it may have a single rule for speed control, five to watch over behaviour at signals, three to watch over behaviour at level crossings...).

Internally each rule comprises one or several DecisionList, although the system is highly flexible and the rules can be programmed in any other way. However, in the interests of simplicity it is recommendable to organise the DecisionList so that each of the rules has a logical defined behaviour.

If the rule watching over the current speed of the train is taken, then following the above reasoning it would be logical to create an initial DecisionList to prepare and set out the conditions for executing the rule, another one to ensure that the current speed does not exceed the maximum permitted speed, another one that displays an on-screen message when the maximum speed is being exceeded and another DecisionList which, for example, saves the maximum 


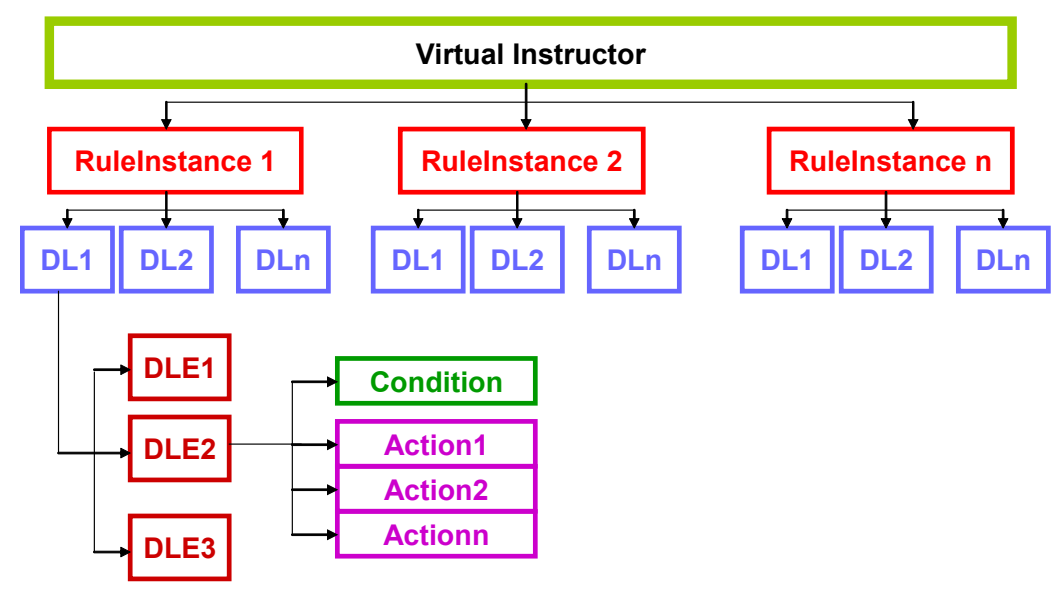

Figure 2.

speed values and the objective speed over time in order to generate a graph for both once the exercise has finished.

Internally each DecisionList is made up of a series of DecisionListEntry, each of which will also be made up of a Condition and a set of Actions.

When the exercise is launched each RuleInstance activates its initial DecisionList (The initial DecisionList is explicitly indicated in the rule configuration file). This initial DecisionList has some characteristics that distinguish it from the other DecisionLists. The two fundamentals are that it activates by default when the exercise is begun and is never deactivated (except at the end of the exercise).

When a DecisionList has been activated the conditions of the DecisionListEntry that are contained in it are evaluated. Depending on the type of DecisionList the evaluation strategy is different, but in all of them, when one of the conditions turns out to be true for the first time, the actions associated with this DecisionListEntry are run (and are marked as run).

As already stated, there are different types of DecisionList, parallel, sequence, one-time and repeat.

Parallel: this is the default DecisionList type, all conditions are tested in parallel and the ordering of conditions inside a list is of no concern.

Sequence: all conditions are tested in parallel, too, but the assessment unit will record whether or not the conditions were fulfilled in the given order.

Repeat: as soon as the list is deactivated, it is activated again

One-time: the conditions are tested only once (this is used for evaluation of the collected assessment parameters)

\subsection{Conditions}

Conditions are those alphanumeric expressions contained in the DecisionListEntries that need to be treated as mathematical expressions; on being evaluated they may give a "true" or "false" result. 
These expressions may include the typical mathematical operators (addition, subtraction, multiplication and division), Boolean operators (and, or, equal, different, greater than, smaller than, greater or equal, smaller or equal) as well as other specific functions devised to simplify the creation of rules.

Below are a few examples of some of the conditions that can be used in 2TRAIN:

SimTrnPosition $==$ sectionBegin

SimTrnPosition $>=$ (levelCrossingPos - relativeStartPos)

SimTrnPosition IN (levelCrossingPos, levelCrossingEndPos)

Or even other more complex ones, such as:

(used(stopPosition) AND (levelCrossingPos $>=$ stopPosition) AND

(SimTrnPosition > stopPosition)) OR (SimTrnPosition >= levelCrossingPos)

\subsection{Actions}

We remind you that actions are those functions that will be run when their associated condition becomes real for the first time. There are actions to assign a particular value to a variable, actions to display on-screen texts, to launch folder viewers, to activate or deactivate a particular DecisionList or even to save data to the AssDB.

Shown below is the structure of some different actions. As can be seen, simplicity has been sought when encoding actions so that staff with no great computer knowledge can create or modify rules.

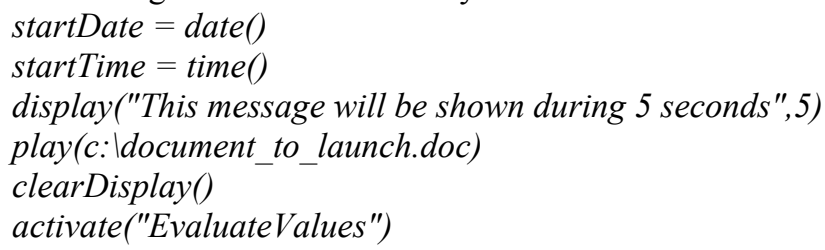

\section{Report}

One of the last objectives of 2TRAIN is to generate a student assessment report. When the exercise is completed all the information referring to the trainee is saved in the database.

Below is shown an outline that the report will follow (this proposal is still not definitive which means the final version may contain minor changes).

As can be seen in the above figures, the report consists of six clearly differentiated parts.

- Heading with general information on the exercise: line, train, and duration of exercise...

- A graph showing the evolution of certain variables over time

- Assessment results in chronological order 


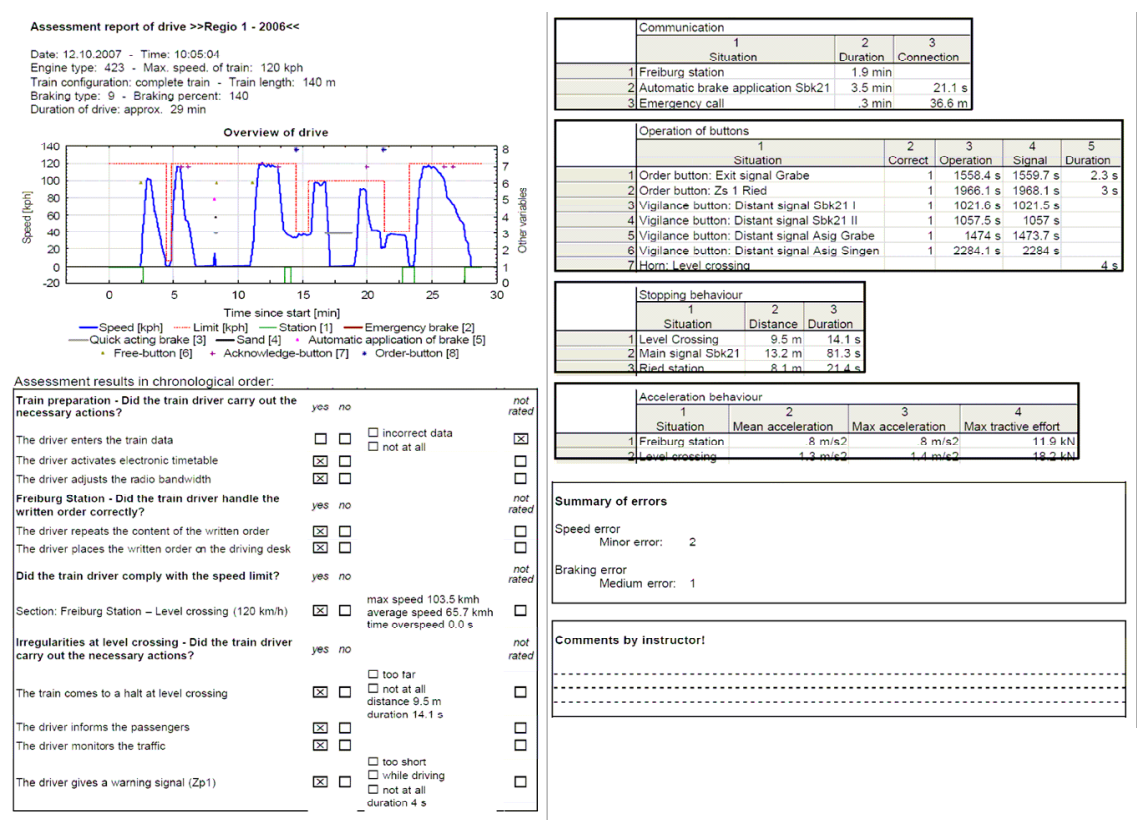

Figure 3.

- Data tables. Data can be entered such as maximum speed, excess speed time, average speed, and time interval between two points...

- Summary of errors in order of importance

- Instructor's comments: free text entered by the instructor.

\section{Conclusions}

The 2TRAIN is a European project whose main objective is to initiate harmonisation in railway train driver training through the use of computer technologies. In order to demonstrate the possibility of achieving this goal, a series of computer tools is being devised which will be connected to three simulators and a CBT.

The programmed software modules include a communication layer compatible with all the simulators (CDSI), an application to help create assessment rules (RBES) and finally, an application to evaluate these rules (VI). A database (AssDB) will also be available with the possibility of a web access where the simulations results will be stored.

Also, thanks to the results of the software demonstration stage previously described and the close collaboration between the different operators taking part in the project, in the 2TRAIN project a series of standards or European recommendations will be drawn up focussed on train driver training. These guides will include particularly detailed guidelines on the safe, efficient and viable use of "computer-based training technologies". 


\section{References}

[1] 2TRAIN Consortium (2008). Benchmarking report training tools and technologies in European railway http:/www.2train.eu/fileadmin/ user_upload/D1.1.1_Catalogue_of_standards_for_training_technology.pdf

[2] 2TRAIN Consortium (2008). Benchmarking report on computer-based railway training in Europe http://www.2train.eu/fileadmin/user_upload/ D1.2.1_Catalogue_of_standardsfor_drivers_competencies_and_training_c ontent.pdf

[3] This report may be found at www.2train.eu in the next few months.

[4] This report may be found at www.2train.eu in the next few months.

[5] http://www.w3.org/XML/

[6] ADO (ActiveX Data Objects)

[7] http://www.corys.com/savoir/simulation.php

[8] Krauss-Maffei Wegmann http://www.kmweg.com/gb/frame.php?page=44

[9] Universitat Passau http://www.uni-passau.de/

[10] http://www.postgresql.org/

[11] Centro de Investigación en Tecnologías Ferroviarias http://www.citef.etsii. upm.es/ 